Abanico Veterinario. Enero-Diciembre 2020; 10:1-10. http://dx.doi.org/10.21929/abavet2020.23

Artículo Original. Recibido: 02/02/2020. Aceptado: 22/07/2020. Publicado: 07/09/2020. Clave: 2020-24.

\title{
El día de parto y tamaño del hato impactan indicadores reproductivos en establos
} familiares

\author{
Calving day of the week and herd size impact reproductive indicators in small-scale \\ dairy farms
}

\section{Montiel-Olguín Luis' ${ }^{1,2}$ ID , Espinosa-Martínez Mario' ${ }^{1 \mathrm{ID}}$, Ruiz-López Felipe ${ }^{1 \mathrm{ID}}$, Estrada-Cortés Eliab ${ }^{3 \mathrm{ID}}$, Durán-Aguilar Marina ${ }^{4}$ ID , Vera-Avila Hector $^{\star 2,4}$ ID}

${ }^{1}$ Centro Nacional de Investigación Disciplinaria en Fisiología y Mejoramiento Animal-INIFAP. México. 2Maestría en Salud y Producción Animal Sustentable, Facultad de Ciencias Naturales, Universidad Autónoma de Querétaro. México. ${ }^{3}$ Campo Experimental Centro Altos de Jalisco-INIFAP. México. ${ }^{4}$ Licenciatura en Medicina Veterinaria y Zootecnia, Facultad de Ciencias Naturales, Universidad Autónoma de Querétaro. México. *Autor responsable y de correspondencia: Vera-Ávila Héctor, Av. de las Ciencias s/n, C.P. 76230 Santiago de Querétaro, Querétaro, México. montiel.luis@inifap.gob.mx, espinosa.mario@inifap.gob.mx, ruiz.felipe@inifap.gob.mx, estrada.eliab@inifap,gob.mx, marina.duran@uaq.mx, hector.raymundo.vera@uaq.mx

\section{Resumen}

El objetivo del presente estudio fue determinar si el día de parto y tamaño del hato impactan el desempeño reproductivo. Durante dieciocho meses se registró información reproductiva proveniente de 23 establos familiares (833 partos) ubicados en Los Altos de Jalisco. Para determinar el efecto de variables independientes (día de parto y tamaño del hato) sobre variables continuas (días a primer servicio (DPS) y días abiertos (DA)) y dicotómicas (asistencia al parto) se utilizaron análisis de varianza y regresión logística, respectivamente. En establos grandes ( $\geq 33$ vacas) no hubo diferencias ( $P>0.05)$ por efecto día de parto sobre DPS. En establos pequeños (<33 vacas), las vacas que parieron en miércoles y jueves tuvieron mayores DPS $(106.1 \pm 10.1$ y $120.8 \pm 11.5(P<0.05)$. Las vacas que parieron en establos grandes en sábado tuvieron mayor cantidad de DA $(136.4 \pm 7.0)$ que cuando los partos se presentaron en otros días $(\mathrm{P}<0.05)$. Finalmente, los establos pequeños tuvieron el doble de asistencias al parto (21.0\%) que los hatos grandes $(10.4 \%)$ durante los fines de semana (viernes a domingo) $(P<0.05)$. En conclusión, los establos grandes se ven menos afectados por el efecto día de parto mientras que los establos pequeños reportan el doble de asistencias al parto durante los fines de semana.

Palabras clave: días a primer servicio, días abiertos, asistencia al parto, lechería familiar.

\begin{abstract}
The objective of the present study was to determine whether the day of the week in which delivery occurs and herd size impact reproductive performance. Reproductive information was recorded for eighteen months from 23 small-scale dairy farms (833 calvings) located in Los Altos of Jalisco state. Analysis of variance and logistic regression analyses were performed to determine the effect of independent variables (day of delivery and herd size) on continuous variables (days at first service (DPS) and days open (DA)) and dichotomous variables (assisted calving), respectively. In big farms ( $\geq 33$ cows) there were no differences $(P>0.05)$ due to delivery day on DPS. In small farms $(<33$ cows $)$, cows that gave birth on Wednesday and Thursday had higher DPS $(106.1 \pm 10.1$ and 120.8 11.5$)(P<0.05)$. Cows that gave birth on Saturday in big farms had higher DA $(136.4 \pm 7.0)$ as compared to those who delivered on other days $(P$ $<0.05)$. Finally, small farms had twice as many assisted calvings $(21.0 \%)$ than large herds $(10.4 \%)$ during
\end{abstract}


weekends (Friday to Sunday) $(P<0.05)$. In conclusion, big farms are less affected by the delivery day effect while small farms reported twice as many assisted calvings during weekends.

Keywords: days at first service, days open, assisted calving, small-scale dairies.

\section{INTRODUCCIÓN}

La producción de leche en el país se lleva a cabo bajo tres sistemas de producción: intensivo, familiar y doble propósito (Vera et al., 2009). El sistema familiar contribuye con una tercera parte de la producción nacional de leche (FAO, 2014), representa poco más del $70 \%$ de los establos lecheros y cuenta con el $20 \%$ del inventario ganadero lechero (Hemme, 2007; Plata-Reyes et al., 2018). Este sistema de producción también contribuye con beneficios sociales, porque mejora la seguridad alimentaria y el estado nutricional de familias en zonas rurales (Hemme y Otte, 2010); además, representa una fuente de ingresos para familias en el campo, lo cual contribuye al arraigo de la población a sus lugares de origen (Espinoza-Ortega et al., 2005). A pesar de la importancia de estos establos, se ha reportado que las unidades de producción operan de manera subóptima, lo cual compromete su sobrevivencia (Posadas-Domínguez et al., 2014; Montiel-Olguín et al., 2019a). Para poder realizar recomendaciones estratégicas y así mejorar su productividad, es necesario hacer un análisis de los factores que impactan negativamente el desempeño en las operaciones de estos establos.

En el sistema familiar, los productores y su familia son el principal soporte operativo de las actividades del establo (García et al., 2012; Jiménez Jiménez et al., 2014). Estas actividades son demandantes en tiempo y tienen alto grado de impacto sobre el desempeño productivo; algunos ejemplos son las labores del campo, la ordeña, alimentación, atender a la recría y el área de partos y la detección de estros (Vera et al., 2009). Por lo tanto, es razonable pensar que debido a la gran cantidad de labores que llevan a cabo los integrantes de la familia, tal vez en algunos días de la semana no se les dedique la misma atención a algunas tareas, por ejemplo, atender correctamente los partos durante los fines de semana. Aunado a esto, en establos del sistema intensivo se ha observado que conforme se incrementa el tamaño de los establos se afecta el desempeño reproductivo (Oleggini et al., 2018; Washburn et al., 2018). También, en el sistema familiar se ha reportado que las vacas en establos más grandes tienen mayor riesgo de requerir asistencia al parto (Montiel-Olguín et al., 2019b).Por lo tanto, en el presente estudio desafiamos la hipótesis de que el día de la semana en el cual ocurre el parto y el tamaño del hato, tienen un impacto sobre los días a primer servicio y los días abiertos como indicadores de desempeño reproductivo.

El objetivo del presente estudio fue determinar si los días de la semana en los cuales ocurre el parto y el tamaño del hato impactan en los indicadores del desempeño reproductivo, en establos bajo el sistema de producción familiar en México. 


\section{MATERIAL Y MÉTODOS}

\section{Ubicación, selección de establos y captura de información}

El estudio se llevó a cabo en la región Los Altos en el estado de Jalisco $\left(20^{\circ} 49^{\prime} 01^{\prime \prime}\right.$ Latitud Norte, 10243'59" Longitud Oeste, elevación 1800 msnm). Se incluyeron 23 establos seleccionados bajo un criterio de muestreo por conveniencia, con las siguientes características; contar con mano de obra familiar como soporte primario en la operación del establo, con menos de 100 vientres en producción, uso de razas especializadas en la producción de leche (Holstein) y con nivel de incorporación de tecnología medio-bajo. Los establos calificaron como característicos del sistema familiar en la región (Vera et al., 2009). El rango en el tamaño de los establos fue entre 24 y 98 vacas; el $99.4 \%$ de las vacas incluidas en el estudio fueron de la raza Holstein.

Durante un periodo de un año y seis meses se realizaron dos visitas por semana a cada unidad de producción y se registró información reproductiva de 833 partos. Durante el periodo de captura se registraron fechas de parto, de servicios, asistencias al parto, retenciones de placenta y diagnósticos de gestación. Los diagnósticos de gestación se llevaron a cabo por palpación transrectal, después de 50 días a partir del último servicio reportado. A partir de la información capturada a nivel de campo, se elaboró una base de datos con la información secuencial de eventos de cada vientre incorporado en el estudio, iniciando a partir de un evento de parto.

\section{Clasificación de variables}

Para la obtención de los indicadores reproductivos días a primer servicio y días abiertos, se utilizaron registros que tuvieran información completa requerida para su cálculo. Además, con fundamentos biológicos y zootécnicos, se decidió depurar registros que tuvieran días a primer servicio menores a 30 y mayores a 278 días de lactación (valor máximo correspondiente al $1 \%$ superior de la muestra), así como días abiertos menores a 30 y mayores a 459 (valor máximo correspondiente al 1\% superior de la muestra). Se calificó como parto con asistencia a aquellos donde se requirió tanto asistencia menor como mayor. Se calificó como retención de placenta cuando las membranas placentarias no se eliminaron antes de las 12 horas post-parto. La clasificación del tamaño de hato se definió con base en la distribución de la muestra por cuartiles (Bijttebier et al., 2017). El primer cuartil correspondió a 32 vacas; por lo tanto, clasificamos establos con $<33$ vacas (pequeños; PQ) y establos $\geq 33$ vacas (medianos y grandes; GD).

\section{Análisis estadístico}

Todos los análisis se llevaron a cabo con el paquete estadístico SAS 9.3 (SAS Institute Inc., Cary, NC). Para determinar el efecto de las variables independientes día de parto y tamaño de hato, sobre las variables cuantitativas discretas días a primer servicio y días abiertos, se utilizó un diseño completamente aleatorizado con el procedimiento GLM. Para la comparación de medias se utilizó la opción PDIFF. Los modelos incluyeron los 
efectos fijos día de la semana en que ocurrió el parto, el tamaño del hato y la interacción. Para determinar el efecto de las variables independientes sobre las variables dicotómicas asistencia al parto, y retención placentaria, se utilizó un modelo de regresión logística, utilizando el procedimiento LOGISTIC. Los modelos incluyeron los efectos día de la semana en que ocurrió el parto, tamaño del hato y la interacción. A partir de los resultados del análisis de regresión logística se obtuvo la razón de momios y sus intervalos de confianza al $95 \%$.

Finalmente se utilizó la prueba de Chi2 con el procedimiento FREQ y la opción CHISQ, para evaluar el efecto de tamaño de hato sobre la presentación de asistencia al parto durante los fines de semana (viernes, sábado y domingo). Para todos los análisis, se consideró como significancia estadística los valores de $\mathrm{P} \leq 0.05$ y como indicador de tendencia a $\mathrm{P} \leq 0.1$.

\section{RESULTADOS Y DISCUSIÓN}

En otros países se ha reportado que el día de la semana impacta algunos eventos reproductivos, como la tasa de concepción (Berry et al., 2011), tasa de asistencias al parto (Mee et al., 2011) y la tasa de inseminación artificial (Tesfaye et al., 2015). Sin embargo, hasta donde tenemos conocimiento, este es el primer reporte sobre el efecto del día de la semana sobre algunos indicadores del desempeño reproductivo, como los días a primer servicio y días abiertos en establos lecheros en México. Los resultados indicaron que, para días a primer servicio y para días abiertos, la interacción Día de parto $X$ Tamaño del hato fue significativa $(P \leq 0.05)$. En el cuadro 1 se muestran las medias ajustadas de la interacción Día de parto $X$ Tamaño de hato para días a primer servicio y días abiertos. Las vacas que parieron en establos pequeños los miércoles y jueves tuvieron mayor cantidad de días a primer servicio (106.1 \pm 10.1 y $120.8 \pm 11.5$, respectivamente), que las vacas que parieron los mismos días en establos grandes (86.6 \pm 4.3 y $87.3 \pm 4.3$, respectivamente). Además, llama la atención que en los establos grandes no hay diferencias $(P>0.05)$, por efecto del día de parto en los días a primer servicio (rango entre $83.9 \pm 4.1-93.2 \pm 4.4$ ). Sin embargo, en establos pequeños, las vacas que parieron en miércoles y jueves tuvieron un número mayor de días a primer servicio (106.1 \pm 10.1 y $120.8 \pm 11.5$ ), comparadas con vacas que parieron en el resto de los días de la semana (rango 70.8 $\pm 11.9-91.0 \pm 11.5)(P \leq 0.05)$.

Respecto a los días abiertos, las vacas que parieron en días sábado y domingo en establos grandes, tuvieron mayor cantidad de días abiertos (136.4 \pm 7.0 y $128.8 \pm 6.5$, respectivamente), en comparación con las vacas que parieron los mismos días en establos pequeños $(89.8 \pm 16.1$ y $98.3 \pm 12.9$, respectivamente) $(P \leq 0.05)$. Por el contrario, las vacas que parieron en jueves en establos grandes, tuvieron menor cantidad de días abiertos (113.4 \pm 6.8$)$, comparadas con las vacas que parieron el mismo día en establos pequeños $(146.4 \pm 18.2)(P \leq 0.05)$. Además, las vacas que parieron en establos grandes en sábado, tuvieron mayor cantidad de días abiertos (136.4 \pm 7.0$)$, que aquellas que 
parieron durante el resto de la semana (rango 112.5 $\pm 7.0-128.8 \pm 6.5)(P \leq 0.05)$; mientras que, en establos pequeños, las vacas que parieron en miércoles y jueves presentaron una mayor cantidad de días abiertos (136.4 \pm 16.1 y $146.4 \pm 18.2$, respectivamente), que aquellas que parieron durante el resto de la semana (rango 89.8 $\pm 16.1-133.8 \pm 18.2$ ) $(\mathrm{P} \leq 0.05)$.

Estos resultados sugieren que en establos grandes del sistema familiar ( $\geq 33$ vacas), el efecto del día de la semana en que ocurrió el parto es menos importante, y debido a esto los días a primer servicio son más homogéneos. Sin embargo, vale la pena resaltar que las vacas que parieron en domingo en establos grandes, presentaron un mayor número de días abiertos. En otros países se ha reportado que las vacas que paren en domingo tienen mayor riesgo de requerir asistencia al parto (Mee et al., 2011); sin embargo, la mortalidad de los recién nacidos no se ve afectada (Mee et al., 2008; Bleul, 2011).

Cuadro 1. Efecto Día de parto X Tamaño de hato para las variables días a primer servicio y días abiertos; medias ajustadas y error estándar

\begin{tabular}{|c|c|c|c|c|}
\hline \multirow[t]{3}{*}{ Día de parto } & \multicolumn{2}{|c|}{ Días a primer servicio } & \multicolumn{2}{|c|}{ Días abiertos } \\
\hline & \multicolumn{2}{|c|}{ Tamaño de hato } & \multicolumn{2}{|c|}{ Tamaño de hato } \\
\hline & GD & $P Q$ & GD & $P Q$ \\
\hline Domingo & $\times 83.9 \pm 4.1$ & $x 82.0 \pm 8.1$ & $x 128.8 \pm 6.5 a$ & $x 98.3 \pm 12.9 b$ \\
\hline Lunes & $x 90.4 \pm 4.3$ & $x 70.8 \pm 11.9$ & $x 124.8 \pm 6.9$ & $x 106.7 \pm 18.9$ \\
\hline Martes & $\times 85.9 \pm 4.4$ & $x 91.0 \pm 11.5$ & $x 112.5 \pm 7.0$ & $x 133.8 \pm 18.2$ \\
\hline Miércoles & $x 86.6 \pm 4.3 a$ & $\mathrm{y} 106.1 \pm 10.1 \mathrm{c}$ & $x 120.5 \pm 6.9$ & $y 136.4 \pm 16.1$ \\
\hline Jueves & $x 87.3 \pm 4.3 a$ & $y 120.8 \pm 11.5 b$ & $x 113.4 \pm 6.8 a$ & $y 146.4 \pm 18.2 c$ \\
\hline Viernes & $x 87.9 \pm 3.9$ & $x 82.6 \pm 12.9$ & $x 118.8 \pm 6.2$ & $x 125.5 \pm 20.6$ \\
\hline Sábado & $x 93.2 \pm 4.4$ & $x 80.5 \pm 10.1$ & $\mathrm{y} 136.4 \pm 7.0 \mathrm{a}$ & $x 89.8 \pm 16.1 b$ \\
\hline
\end{tabular}

Valores con letras diferentes en cada fila para las variables de respuesta días a primer servicio o días abiertos indica diferencia $P \leq 0.05(a, b)$ o tendencia a ser diferentes $P \leq 0.10(a, c)$, respectivamente; Valores con letras diferentes $(x, y)$ dentro de una columna indica diferencia $P \leq 0.05$

Con relación a lo anterior, otro reporte indica que la tasa de preñez se vio disminuida cuando la inseminación artificial se brindó en fines de semana, específicamente en sábado (Berry et al., 2011). Esta información en conjunto sugiere que, en nuestro estudio la mayor tasa de asistencia al parto es resultado en mayor medida por un factor humano y no por cuestiones biológicas. Aunado a esto, la asistencia al parto es un factor de riesgo para días abiertos prolongados en el sistema familiar (Montiel-Olguín et al., 2019a), lo cual, con base en nuestros resultados pudiera estar parcialmente asociado a una mayor contaminación uterina por una atención innecesaria, que prolonga el periodo de puerperio y reduce la tasa de concepción (Sheldon y Owens, 2018). Por otra parte, en otros sistemas de producción se ha reportado que conforme se incrementa el tamaño de los establos se afecta el desempeño reproductivo (Oleggini et al., 2018; Washburn et al., 2018). 
Los establos del sistema familiar son en general más pequeños que los establos con menor número de vacas del sistema intensivo (Val-Arreola et al., 2004). Nuestros resultados sugieren que a pesar de ser establos grandes dentro del sistema familiar, aún pueden ser manejados reproductivamente sin sufrir un efecto negativo por el tamaño del hato, como sucede en los establos intensivos (Oleggini et al., 2018; Washburn et al., 2018). Otro factor que estaría contribuyendo para estos resultados, es el número de personas que trabajan en los hatos familiares. La mano de obra familiar no pagada juega un rol importante para reducir los costos de producción en este sistema de producción (Jiménez Jiménez et al., 2014). Además, se ha reportado que en establos familiares, conforme se incrementa el número de vacas, se contrata a una mayor cantidad de personas ajenas a la familia (Arriaga Jordán et al., 1999; García et al., 2012). Lo anterior explica por qué la atención que se brinda en establos medianos o grandes es más homogénea durante toda la semana, en comparación con lo que ocurre en los establos pequeños.

En el sistema familiar en México, la asistencia al parto y la retención de placenta son factores de riesgo para días a primer servicio tardíos y días abiertos prolongados (MontielOlguín et al., 2019a). Por lo tanto, desafiamos la hipótesis de que el día de la semana en el cual ocurre el parto y el tamaño del hato impactan la proporción de asistencias al parto y de retenciones de placenta. El razonamiento detrás de esta hipótesis, fue que esta condición pudiera estar ocurriendo por fallas en el manejo asociados a los días de descanso (fines de semana), y no por cuestiones biológicas. Referente a asistencia al parto y retención de placenta, los resultados del análisis de regresión logística indicaron que el efecto principal Tamaño del hato fue significativo ( $P \leq 0.05)$; mientras que Día de parto y la interacción Día de parto $X$ Tamaño del hato no $(P>0.1)$. Las vacas que parieron en establos grandes tuvieron una razón de momios de 1.7 para asistencia al parto (IC95\%, 1.01-2.96) y de 1.7 para retención placentaria (IC95\%, 1.04-2.87); es decir, las vacas que paren en establos grandes tienen mayor riesgo de requerir asistencia al parto y presentar retención de placenta, que las vacas que paren en establos pequeños, lo cual ha sido reportado previamente (Mee et al., 2011; Montiel-Olguín et al., 2019a; MontielOlguín et al., 2019b).

En un análisis complementario, encontramos que el tamaño del hato impacta la proporción de asistencias al parto que ocurren durante los fines de semana (viernes, sábado y domingo). Los hatos pequeños tuvieron mayor proporción de asistencias al parto $(21.0 \%)$, que los hatos grandes $(10.4 \%)$ durante esos días $(P \leq 0.05)$. En conjunto, estos resultados sugieren que, aunque los establos grandes reportan en general una mayor cantidad de asistencias al parto y retenciones de placenta, el efecto de los fines de semana impacta en mayor medida a los establos pequeños (el doble de asistencias al parto); esto pudiera ser explicado nuevamente a partir de la cantidad de trabajadores 
disponibles para llevar a cabo las labores durante estos días (Arriaga Jordán et al., 1999; García et al., 2012).

En otros países con sistemas de producción a pequeña escala, se reporta que algunas actividades de importancia reproductiva no se llevan a cabo durante los fines de semana y días feriados, como la inseminación artificial (Tesfaye et al., 2015). Además, es probable que en establos pequeños no haya personal destinado exclusivamente a la tarea de atender partos durante los fines de semana, y aquellos que participan en esta labor tengan menos experiencia y apresuren la atención innecesariamente (Mee et al., 2011). Anteriormente se reportó que el sexo de la cría macho es el principal factor de riesgo, asociado con la asistencia al parto en establos familiares (Montiel-Olguín et al., 2019a). En dicho estudio se plantea que este factor de riesgo desencadena una serie de eventos que repercuten negativamente en el desempeño reproductivo de los establos familiares, colocándolo como un factor clave a controlar (semen sexado como posibilidad). Sin embargo, los hallazgos que aquí reportamos colocan a la atención al parto como otro factor clave, el cual pudiera ser independiente y aditivo del componente biológico (sexo de la cría macho). Una recomendación práctica que se desprende de estos últimos resultados, es que en los programas de extensionismo, se debería enfatizar la importancia de la atención a los partos en hatos pequeños del sistema familiar (específicamente durante los fines de semana).

\section{CONCLUSIONES}

El día de la semana en el cual ocurre el parto y el tamaño del hato, impactan los días a primer servicio, días abiertos y las tasas de asistencia en vacas bajo el sistema de producción familiar. En establos grandes ( $\geq 33$ vacas), los promedios de días a primer servicio y días abiertos son más homogéneos durante toda la semana, en comparación con los valores en establos pequeños (<33 vacas). Asimismo, las vacas en los establos pequeños tienen una mayor proporción de asistencias al parto durante los fines de semana, esto probablemente asociado a fallas en la atención de los partos durante los días de descanso.

\section{AGRADECIMIENTOS}

Fondo INIFAP recursos fiscales SIGI 15352034772.

\section{LITERATURA CITADA}

ARRIAGA JORDÁN CM, Espinosa Ortega A, Rojo Guadarrama H, Valdéz Martínez JL, Sánchez Vera E, Wiggins S. 1999. Aspectos socio-económicos de la producción campesina de leche en el valle de Toluca: I. evaluación económica inicial. Agrociencia. 33(4):483-492. ISSN-e 1405-319. http://www.colpos.mx/agrocien/9699/334.pdf 
BERRY DP, Evans, RD, Mc Parland S. 2011. Evaluation of bull fertility in dairy and beef cattle using cow field data. Theriogenology. 75(1):172-181. https://doi.org/10.1016/j.theriogenology.2010.08.002

BIJTTEBIER J, Hamerlinck J, Moakes S, Scollan N, Van Meensel J, Lauwers L. 2017. Low-input dairy farming in Europe: Exploring a context-specific notion. Agricultural Systems. 156(Suppl C):43-51. https://doi.org/10.1016/j.agsy.2017.05.016

BLEUL U. 2011. Risk factors and rates of perinatal and postnatal mortality in cattle in Switzerland. Livestock Science. 135(2-3):257-264. https://doi.org/10.1016/j.livsci.2010.07.022

ESPINOZA-ORTEGA A, Álvarez-Macías A, del Valle MDC, Chauvete M. 2005. La economía de los sistemas campesinos de producción de leche en el Estado de México. Técnica Pecuaria México. 43(1):39-56. ISSN: 0040-1889. https://cienciaspecuarias.inifap.gob.mx/index.php/Pecuarias/article/view/1393

FAO. FOOD AND AGRICULTURE ORGANIZATION OF THE UNITED NATIONS. 2014. Agricultura Familiar en América Latina y el Caribe: Recomendaciones de Política. Chile. 2014. ISBN 978-92-5-308364-0 http://www.fao.org/3/i3788s/i3788s.pdf

GARCÍA CGM, Dorward P, Rehman T. 2012. Farm and socio-economic characteristics of smallholder milk producers and their influence on technology adoption in Central Mexico. Tropical Animal Health and Production. 44:1199-1211. https://doi.org/10.1007/s11250011-0058-0

HEMME T, Otte J. 2010. Status and prospects for smallholder milk production: a global perspective. Rome: Food and Agriculture Organization of the United Nations (FAO). Pp. 181. ISBN 978-92-5-106545-7 http://www.fao.org/3/i1522e/i1522e.pdf

HEMME T. IFCN (Dairy Team and IFCN Researchers) 2007. IFCN Dairy Report 2007, International Farm Comparison Network. IFCN Dairy Research Center, Kiel, Germany. https://ifcndairy.org/publications/

JIMÉNEZ JIMÉNEZ RA, Espinosa Ortiz V, Soler Fonseca DM. 2014. El costo de oportunidad de la mano de obra familiar en la economía de la producción lechera de Michoacán, México. Revista de Investigación Agraria y Ambiental. 5(1):47-56. ISSN 21456097. https://dialnet.unirioja.es/descarga/articulo/5344972.pdf 
MEE JF, Berry DP, Cromie AR. 2008. Prevalence of, and risk factors associated with, perinatal calf mortality in pasture-based Holstein-Friesian cows. Animal. 2(4):613-620. https://doi.org/10.1017/S1751731108001699

MEE JF, Berry DP, Cromie AR. 2011. Risk factors for calving assistance and dystocia in pasture-based Holstein-Friesian heifers and cows in Ireland. The Veterinary Journal. 187(2):189-194. https://doi.org/10.1016/j.tvjl.2009.11.018

MONTIEL-OLGUÍN LJ, Estrada-Cortés E, Espinosa-Martínez MA, Mellado M, Hernández-Vélez JO, Martínez-Trejo G, Ruiz-López FJ, Vera-Avila HR. 2019a. Risk factors associated with reproductive performance in small-scale dairy farms in Mexico. Tropical Animal Health and Production. 51(1):229-236. https://doi.org/10.1007/s11250018-1681-9

MONTIEL-OLGUÍN LJ, Estrada-Cortés E, Espinosa-Martínez MA, Mellado M, Hernández-Vélez JO, Martínez-Trejo G, Hernández-Andrade L, Hernández-Ortíz R, Alvarado-Islas A, Ruiz-López FJ, Vera-Avila HR. 2019b. Factores de riesgo a nivel de establo asociados con el desempeño reproductivo en el sistema de producción de leche a pequeña escala en México. Revista Mexicana de Ciencias Pecuarias. 10(3):676-691. https://doi.org/10.22319/rmcp.v10i3.4825

OLEGGINI GH, Ely LO, Smith JW. 2018. Effect of region and herd size on dairy herd performance parameters. Journal of Dairy Science. 84(5):1044-1050. https://doi.org/10.3168/jds.S0022-0302(01)74564-X

PLATA-REYES DA, Morales-Almaraz E, Martínez-García CG, Flores-Calvete G, LópezGonzález F, Prospero-Bernal F, Valdez-Ruiz CL, Zamora Juarez YG, Arriaga-Jordán CM. 2018. Milk production and fatty acid profile of dairy cows grazing four grass species pastures during the rainy season in small-scale dairy systems in the highlands of Mexico. Tropical Animal Health and Production. 50(8):1797-1805. https://doi.org/10.1007/s11250-018-1621-8

POSADAS-DOMÍNGUEZ RR, Arriaga-Jordán CM, Martínez-Castañeda FE. 2014. Contribution of family labour to the profitability and competitiveness of small-scale dairy production systems in central Mexico. Tropical Animal Health and Production. 46(1):235240. https://doi.org/10.1007/s11250-013-0482-4

SHELDON IM, Owens SE. 2018. Postpartum uterine infection and endometritis in dairy cattle. Animal Reproduction. 14(3):622-629. http://dx.doi.org/10.21451/1984-3143AR1006 
TESFAYE A, Alemayehu L, Tefera Y, Endris A. 2015. Factors affecting the reproductive performance of smallholder dairy cows in two regions of Ethiopia. Livestock Research for Rural Development. 27. http://www.lrrd.org//rrd27/3/tesf27046.html

VAL-ARREOLA D, Kebreab E, Dijkstra J, France J. 2004. Study of the lactation curve in dairy cattle on farms in central Mexico. Journal of Dairy Science. 87(11):3789-3799. https://doi.org/10.3168/jds.S0022-0302(04)73518-3

VERA AH, Hernández AL, Espinosa GJ, Ortega RL, Díaz AE, Ponce HR, Núñez HG, Medina CM, Ruiz LF. 2009. Producción de leche de bovino en el sistema familiar. INIFAP. CIRGOC. Libro Técnico Núm. 24. Veracruz, México. 384p. ISBN 978-607-425-269-9. http://biblioteca.inifap.gob.mx:8080/jspui/handle/123456789/1563

WASHBURN SP, Silvia WJ, Brown CH, McDaniel BT, McAllister AJ. 2018. Trends in reproductive performance in Southeastern Holstein and Jersey DHI herds. Journal of Dairy Science. 85(1):244- 251. https://doi.org/10.3168/jds.S0022-0302(02)74073-3 